\title{
Matrix model and Yukawa couplings on the noncommutative torus
}

\author{
Masaki Honda \\ Department of Physics, Waseda University, \\ Tokyo 169-8555, Japan \\ E-mail: yakkuru_111@ruri.waseda.jp
}

ABSTRACT: The IKKT model is proposed as a non-perturbative formulation of superstring theory. We propose a Dirac operator on the noncommutative torus, which is consistent with the IKKT model, based on noncommutative geometry. Next, we consider zero-mode equations of the Dirac operator with magnetic fluxes. We find that zero-mode solutions have the chirality and the generation structures similar to the commutative case. Moreover, we compute Yukawa couplings of chiral matter fields.

Keywords: M(atrix) Theories, Compactification and String Models, Non-Commutative Geometry

ARXIV EPRINT: 1901.00095 


\section{Contents}

1 Introduction 1

2 IKKT model $\quad 3$

2.1 Ishibashi-Kawai-Kitazawa-Tsuchiya (IKKT) model 3

$2.2 \mathrm{NC}$ torus in the IKKT model 4

3 Toroidal compactifications of 10D SYM theory $\quad 6$

$\begin{array}{lll}3.1 & \text { Twisted bundle on the torus } & 6\end{array}$

$\begin{array}{lll}3.2 & \text { Zero-modes of Dirac operator on } T^{2} & 7\end{array}$

4 Dirac operator on the NC torus and the IKKT model 9

4.1 Differential operators on the NC torus 9

4.2 Dirac operator on the NC torus based on the IKKT model 9

5 Zero-mode analysis on the magnetized NC torus 11

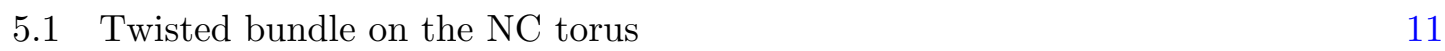

5.2 Zero-modes of the Dirac operator $\not_{\text {phys }} \quad 12$

$\begin{array}{ll}5.3 \text { Eigenvalues of the Laplacian } & 14\end{array}$

$\begin{array}{lll}5.4 & \text { Normalizations and Yukawa couplings } & 15\end{array}$

$\begin{array}{llr}6 & \text { Conclusions and discussions } & 19\end{array}$

$\begin{array}{ll}\text { A Effective action of the IKKT model } & 21\end{array}$

\section{Introduction}

Superstring theory is a promising candidate for a unified theory of all forces in nature. Superstring theory is only defined perturbatively and has infinite degenerate vacua. Therefore, it is said that superstring theory has no predictions for our world, and we need a more fundamental theory.

Matrix models are proposed as a non-perturbative formulation of superstring theory. In this paper, we focus on Ishibashi-Kawai-Kitazawa-Tsuchiya (IKKT) model [1]. This model is derived from the matrix regularization of the Green-Schwarz action in the Schild gauge or large-N reduced model of ten-dimensional (10D) super Yang-Mills (SYM) theory.

In the IKKT model, matter fields and degree of freedom of spacetime are considered to be embedded in matrices. Several attempts have been made to show it. In ref. [2], the authors considered an intersecting flat D-branes system based on the IKKT model. To analyze the chirality and the generation structures of the system, they used the analogy of the harmonic oscillator in quantum mechanics. They showed the existence of a chiral 
zero-mode which are coming from a string connecting two different D-branes. However, they considered a simple configuration, e.g., two D-branes are orthogonal. Therefore, they could not realize multiple chiral zero-modes. In addition, in more general configurations, we cannot easily find the chiral zero-modes by using the same method because the complicated contribution comes from the mixing term between two D-branes. In ref. [3], the authors considered intersecting fuzzy spheres as compact D-branes and realized two chiral zeromodes. To admit a fuzzy sphere as a classical solution, we must introduce a new term. However, the origin of such a term is not clear. In either case, it is difficult to obtain the number of chiral zero-modes that we expect. In ref. [4], the authors challenged the realization of three generations by using numerical analysis. By considering the squashed fuzzy sphere in addition to the fuzzy spheres, they succeeded in realizing three generations numerically. On the other hand, the authors of ref. [5], considered a fuzzy torus with a magnetic flux based on the finite-matrix formulation of gauge theories. ${ }^{1}$ They computed the index of the overlap Dirac operator on the fuzzy torus by the Monte Carlo simulations and showed the numerical results that are consistent with the index theorem. In this formulation, the exact relationship with the IKKT model is not clear because we must introduce the special type of the Dirac operator to obtain the non-trivial index of the Dirac operator. Moreover, it is still difficult to compute physically important quantities like Yukawa couplings.

The purposes of this paper are to define a Dirac operator that is consistent with the IKKT model, to analyze the chirality and the generation structures and to compute Yukawa couplings of chiral matter fields.

To analyze concretely, we consider the noncommutative (NC) torus as a classical solution of the IKKT model. It is known that the NC torus as an irrational rotation ring can be realized in the IKKT model [6]. In addition, we consider the analogy of the toroidal compactifications of 10D SYM theory. In ref. [7], the authors considered the toroidal compactifications of 10D SYM theory with magnetic fluxes. A key concept is the twisted bundle. The twisted bundle can be interpreted as a compatibility condition between the periodic boundary conditions on the torus and the gauge transformations. This compatibility condition implies that the magnetic fluxes are quantized, then the zero-mode solution of the Dirac equation can have the chirality and the degeneracies. The authors of ref. [7] identified these results as the chirality and the generation structures in the four-dimensional effective theory and computed the Yukawa couplings by computing the overlap integral over the torus. From the viewpoint of phenomenology in superstring theory, this result is very important. Therefore, we consider the twisted bundle on the NC torus.

In the following, we propose a Dirac operator on the NC torus that is consistent with the IKKT model, and we solve the zero-mode equation of this Dirac operator with magnetic fluxes. Then, we compute the normalization factors of the zero-mode solutions and Yukawa couplings of chiral matter fields.

\footnotetext{
${ }^{1}$ In this paper, we use noncommutative to describe infinite dimensional representations or operator algebras and fuzzy to describe finite dimensional representations or approximations.
} 
The organization of this paper is as follows. In section 2, we briefly review the IKKT model and the realization of the NC torus in the IKKT model. In section 3, we review the basic results of the toroidal compactifications of 10D SYM theory. In section 4, we introduce differential operators on the NC torus based on noncommutative geometry. In addition, we propose a Dirac operator that is consistent with the IKKT model. In section 5, we solve the zero-mode equation by using the analogy of the Fourier transformation. In addition, we compute the normalization factors of the zero-mode solutions and the Yukawa couplings of chiral matter fields. To compute, we define the trace that is consistent with the gauge transformations and the torus translations. Section 6 contains conclusions and discussion.

\section{IKKT model}

\subsection{Ishibashi-Kawai-Kitazawa-Tsuchiya (IKKT) model}

The action of the IKKT model is defined as follows [1],

$$
S=-\frac{1}{g^{2}} \operatorname{Tr}\left(\frac{1}{4}\left[X_{M}, X_{N}\right]\left[X^{M}, X^{N}\right]+\frac{1}{2} \bar{\psi} \Gamma^{M}\left[X_{M}, \psi\right]\right),
$$

where $X^{M}(M=0 \sim 9)$ is a $10 \mathrm{D}$ vector and $N \times N$ Hermitian matrix, $\psi$ is a 10D MajoranaWeyl spinor whose components are $N \times N$ matrices and $g$ is a scale factor. Indices are contracted by the Minkowski metric. On the other hand, we will see that this action admits an infinite dimensional representation like linear operators in the next subsection.

The action (2.1) has some symmetries:

$$
\begin{aligned}
\delta^{(1)} X^{M} & =i \bar{\epsilon}_{1} \Gamma^{M} \psi, & \delta^{(1)} \psi & =\frac{i}{2} \Gamma^{M N}\left[X_{M}, X_{N}\right] \epsilon_{1}, \\
\delta^{(2)} X^{M} & =0, & \delta^{(2)} \psi & =\epsilon_{2} 1_{N}, \\
\delta_{T} X^{M} & =c^{M} 1_{N}, & \delta_{T} \psi & =0, \\
\delta_{G} X^{M} & =i\left[\Lambda, X^{M}\right], & \delta_{G} \psi & =i[\Lambda, \psi],
\end{aligned}
$$

where $\epsilon_{i}(i=1,2)$ is $10 \mathrm{D}$ Majorana-Weyl spinor as a Grassmann odd parameter, $c^{M}$ is a $10 \mathrm{D}$ constant vector, $1_{N}$ is the $N \times N$ identity matrix, and $\Lambda$ is a $N \times N$ Hermitian matrix.

Eqs. (2.2) and (2.3) can be identified $\mathcal{N}=2$ supersymmetry (SUSY) as follows. If we consider a linear combination of $\delta^{(1)}$ and $\delta^{(2)}$ as

$$
\tilde{\delta}^{(1)} \equiv \delta^{(1)}+\delta^{(2)}, \quad \tilde{\delta}^{(2)} \equiv i\left(\delta^{(1)}-\delta^{(2)}\right),
$$

then we can obtain

$$
\left[\tilde{\delta}_{\epsilon}^{(i)}, \tilde{\delta}_{\xi}^{(j)}\right] X^{M}=2 i \bar{\epsilon} \Gamma^{M} \xi \delta^{i j}, \quad\left[\tilde{\delta}_{\epsilon}^{(i)}, \tilde{\delta}_{\xi}^{(j)}\right] \psi=0 \quad(i, j=1,2),
$$

where we used eq. (2.5) and the equation of motion of $\psi$. If we identify $X^{M}$ as a 10D spacetime coordinate, eq. (2.6) is $\mathcal{N}=2$ on-shell SUSY algebra. In this sense, we often say that the degree of freedom of spacetime is embedded in matrices. From this, there is a possibility of analysis for the dynamics of spacetime, e.g., a mechanism of compactifications. 
When $\psi=0$, the equation of motion of $X^{M}$ is

$$
\left[X^{N},\left[X^{M}, X^{N}\right]\right]=0 .
$$

The simplest solution is ${ }^{\forall}\left[X^{M}, X^{N}\right]=0$. However, we can show that attractive forces act between the eigenvalues in the one-loop effective potential around this vacuum. Therefore, they do not spread, and the correspondence with the original theory does not hold. In this case, we need more conditions on gauge groups $[8,9]$.

The second simplest, however, an interesting solution is that some commutators are proportional to the identity matrix, i.e.,

$$
\left[X^{M}, X^{N}\right]=i \theta^{M N},
$$

where $\theta^{M N}$ is a real anti-symmetric matrix with respect to the Lorentz indices. We omit the identity matrix. When each matrix has a finite size, eq. (2.8) is a contradiction. We should interpret eq. (2.8) as it is satisfied at large matrix size. This is the correspondence between a function algebra and a matrix algebra in the matrix regularization. On the other hand, the infinite dimensional representation, which we are interested, strictly respects eq. (2.8).

We can also interpret eq. (2.8) as a D-branes (BPS) configuration as follows. We can find that the transformation by $\delta^{(1)}$ is proportional to the identity matrix and cancel the transformation by $\delta^{(2)}$. Explicitly, if we set $\epsilon_{2}= \pm \frac{1}{2} \theta^{M N} \Gamma_{M N} \epsilon_{1}$, then

$$
\left(\delta^{(1)} \pm \delta^{(2)}\right) X^{M}=0, \quad\left(\delta^{(1)} \pm \delta^{(2)}\right) \psi=0 .
$$

Therefore, the half of $\mathcal{N}=2$ SUSY is preserved in this background, and the one-loop effective potential exactly vanishes due to SUSY. It is an advantage of this model that a many-body system of D-branes can be realized. When we consider two sets of eq. (2.8),

$$
\left[X_{(1)}^{M}, X_{(1)}^{N}\right]=i \theta_{(1)}^{M N}, \quad\left[X_{(2)}^{M}, X_{(2)}^{N}\right]=i \theta_{(2)}^{M N},
$$

then

$$
X^{M}=\left(\begin{array}{cc}
X_{(1)}^{M} & 0 \\
0 & X_{(2)}^{M}
\end{array}\right)
$$

is also a solution of eq. (2.7). In general, eq. (2.9) is not a D-branes configuration. However, some configurations become a D-branes configuration again, e.g., the case of $X_{(1)}^{M}=X_{(2)}^{M}$ is interpreted as two coincident D-branes, and other cases are considered in ref. [1].

\subsection{NC torus in the IKKT model}

In this subsection, we briefly review the NC torus in the IKKT model based on refs. [6, 10]. A unitary transformation is defined by

$$
X^{M} \rightarrow U X^{M} U^{-1}, \quad \psi \rightarrow U \psi U^{-1},
$$

where $U$ is a unitary matrix (operator). If the trace in the action (2.1) has the cyclic property, this unitary transformation becomes a symmetry of the IKKT model. The infinitesimal form of eq. (2.10) corresponds to eq. (2.5). The authors of ref. [6] showed that the NC torus can be realized by this unitary transformation. 
We restrict the action (2.1) to the subspace of $\left(X^{M}, \psi\right)$ belonging to the same gauge class before and after translations in the directions $X^{4}$ and $X^{5}$. In other word, we consider conditions as follows,

$$
\begin{aligned}
U_{4} X^{4} U_{4}^{-1} & =X^{4}+2 \pi R_{4}, & & U_{5} X^{5} U_{5}^{-1}=X^{5}+2 \pi R_{5}, \\
U_{i} X^{M} U_{i}^{-1} & =X^{M} & & (i \neq M, i=4,5, M=0 \sim 9), \\
U_{i} \psi U_{i}^{-1} & =\psi & & (i=4,5),
\end{aligned}
$$

where $R_{i}(i=4,5)$ is the scalar matrix (operator) of a real coefficient corresponding to the periods of the torus. In the following, let us define $M=0 \sim 9, \mu=0 \sim 3$ and $i=4 \sim 9$.

We can see that any finite dimensional representations do not satisfy the conditions (2.11). However, we can find solutions if we interpret $X^{M}$ and $\psi$ as operators on an infinite dimensional Hilbert space. ${ }^{2}$

From eq. (2.11), we can confirm that $U_{4} U_{5} U_{4}^{-1} U_{5}^{-1}$ commutes with $X^{M}$ and $\psi$. Therefore, we assume (or we may be able to apply Schur's lemma for countable dimensional cases $[11,12]$ because we can also realize $U_{4}$ and $U_{4}$ as operators on $\left.l^{2}(\mathbb{R})\right)$ that $U_{4} U_{5} U_{4}^{-1} U_{5}^{-1}$ is a scalar operator, i.e.,

$$
U_{4} U_{5}=e^{2 \pi i \theta} U_{5} U_{4},
$$

where $\theta$ is a real parameter. Eq. (2.12) is the algebra of the $\mathrm{NC}$ torus in mathematics. Precisely, we can restrict $\theta \in[0,1 / 2]$ because of some isomorphisms. Depending on whether the parameter $\theta$ is a rational number or an irrational number, the mathematical structure differs. In fact, the algebra of the $\mathrm{NC}$ torus admits a finite dimensional representation if $\theta \in \mathbb{Q}$, and we cannot realize such algebra by the finite dimensional representation if $\theta \in \mathbb{R} \backslash \mathbb{Q}$. In this paper, we assume $\theta \in \mathbb{R} \backslash \mathbb{Q}$ because we are interested in the infinite dimensional representation of the $\mathrm{NC}$ torus. In this sense, $X^{M}, \psi, U_{4}$ and $U_{5}$ are no longer finite dimensional matrices but operators. We use "hat" to indicate that it is an operator (we omit the identity operator).

In the following, we show a concrete representation of $\hat{X}^{M}, \hat{U}_{4}$ and $\hat{U}_{5}$. Let us start from a Hilbert space $\mathcal{H}=L^{2}(\mathbb{R}) \otimes \mathbb{C}^{m}, m \in \mathbb{N}\left(m\right.$ corresponds to $\mathbb{Z}_{m}$ in the usual construction $[6,10,13])$. We can realize $\hat{U}_{4}$ and $\hat{U}_{5}$ as operators on $\mathcal{H}$, i.e.,

$$
\hat{U}_{4}|x: j\rangle=\left|x-\frac{n}{m}+\theta: j-1\right\rangle, \quad \hat{U}_{5}|x: j\rangle=\exp \left[-2 \pi i\left(x-\frac{n j}{m}\right)\right]|x: j\rangle,
$$

where $n$ is some integer. For simplicity, we assume that $m$ and $n$ are positive and co-prime each other (or $m=1$ and $n=0$ ). We define $\hat{X}^{4}$ and $\hat{X}^{5}$ as

$$
\hat{X}^{4}:=\frac{2 \pi m}{n-m \theta} R_{4} \hat{x} \otimes 1_{m}, \quad \hat{X}^{5}:=R_{5} \hat{p} \otimes 1_{m},
$$

where $1_{m}$ is the $m \times m$ identity matrix, $\hat{x}$ and $\hat{p}$ are the position operator and the momentum operator on one-dimensional quantum mechanics (where $\hbar=1$ ), respectively. We can see

\footnotetext{
${ }^{2}$ In general, the trace on an infinite dimensional Hilbert space does not satisfy the cyclic property (e.g., the position operators, the momentum operators and their commutation relations in quantum mechanics). We will define the trace which is consistent with the gauge transformations (2.10) and the conditions (2.11).
} 
that $\theta^{45}=\frac{2 \pi m}{n-m \theta} R_{4} R_{5}$ and easily confirm that eqs. (2.11) and (2.12) are satisfied. In the following, since the part acting on $\mathbb{C}^{m}$ is the identity matrix, we use the Hilbert space spanned by $\left\{\left|X^{4}\right\rangle ; \hat{X}^{4}\left|X^{4}\right\rangle=X^{4}\left|X^{4}\right\rangle\right\}$ or $\left\{\left|X^{5}\right\rangle ; \hat{X}^{5}\left|X^{5}\right\rangle=X^{5}\left|X^{5}\right\rangle\right\}$.

From an analogy of quantum mechanics,

$$
\left\langle X^{4} \mid X^{5}\right\rangle=\frac{1}{A} \exp \left[i \frac{X^{4} X^{5}}{\theta^{45}}\right], \quad A=(2 \pi)^{2} R_{4} R_{5} \quad \text { (the area of the torus) }
$$

since the periodicity is realized by the unitary operators $\hat{U}_{4}$ and $\hat{U}_{5}$, and we restrict the fundamental (or physical) region to $0 \leq X^{4} \leq 2 \pi R_{4}$ and $0 \leq X^{5} \leq 2 \pi R_{5}{ }^{3}$

\section{Toroidal compactifications of 10D SYM theory}

In this section, we review the basic results of toroidal compactifications of 10D SYM theory based on refs. [7, 14].

\subsection{Twisted bundle on the torus}

Let us start from $\mathrm{U}(1)$ gauge theory on the torus $T^{2}$. In this paper, we take a lattice $\Lambda \simeq \mathbb{Z}^{2}$ that is generated by $v^{1}=\left(2 \pi R_{4}, 0\right)$ and $v^{2}=\left(0,2 \pi R_{5}\right)$, and $T^{2}$ is defined by $\mathbb{R}^{2} / \Lambda$.

A constant magnetic flux is introduced by

$$
A_{4}=0, \quad A_{5}=F x^{4}
$$

then the magnetic flux $F_{45}=F$. We use the same gauge choice (axial gauge in ref. [14]) in section 5 .

We must confirm that the gauge theory is well-defined on $T^{2}$. Obviously, we should confirm only about $x^{4}$-direction. The covariant derivatives transform like

$$
D_{4} \rightarrow D_{4}, \quad D_{5} \rightarrow D_{5}-2 \pi i R_{4} F
$$

If this translation can be absorbed as the gauge symmetry, $\mathrm{U}(1)$ gauge theory on $T^{2}$ is well-defined. Actually, we can realize eq. (3.2) by

$$
\Omega_{4}\left(x^{4}, x^{5}\right) D_{4} \Omega_{4}^{-1}\left(x^{4}, x^{5}\right)=D_{4}, \quad \Omega_{4}\left(x^{4}, x^{4}\right) D_{5} \Omega_{4}^{-1}\left(x^{4}, x^{5}\right)=D_{5}-2 \pi i R_{4} F,
$$

where $\Omega_{4}\left(x^{4}, x^{5}\right)=\exp \left[2 \pi i R_{4} F x^{5}\right]$. In general, we need to consider another gauge transformation $\Omega_{5}\left(x^{4}, x^{5}\right)$ associated with the translation along the $x^{5}$-direction. In the above case, $\Omega_{5}\left(x^{4}, x^{5}\right)=1$. The above concept is called the twisted bundle.

In addition, we must confirm a consistency condition

$$
\Omega_{5}\left(x^{4}+2 \pi R_{4}, x^{5}\right) \Omega_{4}\left(x^{4}, x^{5}\right)=\Omega_{4}\left(x^{4}, x^{5}+2 \pi R_{5}\right) \Omega_{5}\left(x^{4}, x^{5}\right) .
$$

\footnotetext{
${ }^{3}$ Let $\mathcal{H}_{m}(m \in \mathbb{Z})$ be a Hilbert space such that the spectrum of the Hermitian operator $\hat{X}^{4}$ lies in the interval $2 \pi m R_{4} \leq X^{4} \leq 2 \pi(m+1) R_{4}$. In this case, $\mathcal{H}_{m}$ is unitary equivalent to ${ }^{\forall} \mathcal{H}_{n \in \mathbb{Z}}$ Therefore, we interpret the fundamental (or physical) region with respect to the $X^{4}$-direction is $0 \leq X^{4} \leq 2 \pi R_{4}$. We interpret the $X^{5}$-direction as well.
} 
Eq. (3.3) implies

$$
\frac{A}{2 \pi} F \in \mathbb{Z},
$$

where $A=(2 \pi)^{2} R_{4} R_{5}$ is the area of the torus. Namely, the magnetic flux on the torus is quantized.

\subsection{Zero-modes of Dirac operator on $T^{2}$}

We can analytically solve the zero-mode equations of the Dirac operator with the background gauge field (3.1). The Dirac operator is defined by

$$
\not D=\sum_{i=4,5} \sigma_{i} D_{i}=\left(\begin{array}{cc}
0 & \partial_{4}-i \partial_{5}-F x^{4} \\
\partial_{4}+i \partial_{5}+F x^{4} & 0
\end{array}\right),
$$

where $\sigma_{4}$ and $\sigma_{5}$ are the Pauli matrices $\sigma_{1}$ and $\sigma_{2}$, respectively. For convenience, we rewrite the constant magnetic flux as $F=\frac{2 \pi}{A} N \nu$, where $N$ is a positive integer and $\nu=\operatorname{sign}(F)$. The zero-mode equation of the Dirac operator is

$$
\not D \psi=0 .
$$

If $\psi$ is labeled by the eigenvalue of the chirality matrix $\sigma_{3}$, i.e.,

$$
\psi=\left(\begin{array}{l}
\psi^{+} \\
\psi^{-}
\end{array}\right)
$$

the zero-mode equations of each component can be written as

$$
\left(\partial_{4}+i s \partial_{5}+s F x^{4}\right) \psi^{s}=0 \quad(s= \pm 1) .
$$

We can easily factorize $\psi^{s}$ satisfying eq. (3.6) as

$$
\psi^{s}\left(x^{4}, x^{5}\right)=\exp \left[-\frac{1}{2} s F\left(x^{4}\right)^{2}\right] f^{s}\left(x^{4}+i s x^{5}\right),
$$

where $f^{s}\left(x^{4}+i s x^{5}\right)$ satisfies

$$
\begin{aligned}
f^{s}\left(x^{4}+2 \pi R_{4}+i s x^{5}\right) & =\exp \left[\frac{N}{R_{5}} s \nu\left(x^{4}+i s x^{5}+\pi R_{4}\right)\right] f^{s}\left(x^{4}+i s x^{5}\right), \\
f^{s}\left(x^{4}+i s\left(x^{5}+2 \pi R_{5}\right)\right) & =f^{s}\left(x^{4}+i s x^{5}\right) .
\end{aligned}
$$

$f^{s}\left(x^{4}+i s x^{5}\right)$ is periodic with respect to the $x^{5}$-direction. Therefore, we can use the Fourier expansion as

$$
f^{s}\left(x^{4}+i s x^{5}\right)=\sum_{n \in \mathbb{Z}} C_{n}^{s} \exp \left[\frac{n s}{R_{5}}\left(x^{4}+i s x^{5}\right)\right] .
$$

From the first condition of eq. (3.7), the Fourier coefficients satisfy

$$
C_{n}^{s}=\exp \left[\frac{s \pi R_{4}}{R_{5}}(N \nu-2 n)\right] C_{n-N \nu}^{s} .
$$


If we decompose $n$ into $n=N p+q(p \in \mathbb{Z}, q=0, \ldots, N-1)$, thus we can rewrite eq. (3.8) as

$$
C_{N p+q}^{s}=\exp \left[-\frac{\pi R_{4}}{R_{5}} s \nu\left(N p^{2}+2 p q\right)\right] C_{q}^{s} .
$$

From the above, $f^{s}\left(x^{4}+i s x^{5}\right)$ can be written as

$$
f^{s}\left(x^{4}+i s x^{5}\right)=\sum_{q=0}^{N-1} C_{q}^{s} \sum_{p \in \mathbb{Z}} \exp \left[-\frac{\pi R_{4}}{R_{5}} s \nu\left(N p^{2}+2 p q\right)\right] \exp \left[\frac{s}{R_{5}}(N p+q)\left(x^{4}+i s x^{5}\right)\right] .
$$

If $\nu s=+1, f^{s}\left(x^{4}+i s x^{5}\right)$ converges. In other words, the sign of the magnetic flux determines the chirality of the zero-mode solution. In addition, the summation with respect to $q$ means that there are $N$-independent zero-mode solutions. In ref. [7], these results are interpreted as the chirality and the generation structures in the four-dimensional effective theory.

Therefore, each degenerated solution of eq. (3.6) is

$$
\begin{aligned}
& \psi_{q}^{s}\left(x^{4}, x^{5}\right)= \\
& C_{q}^{s} \exp \left[-\frac{|F|}{2}\left(x^{4}\right)^{2}\right] \sum_{p \in \mathbb{Z}} \exp \left[-\frac{\pi R_{4}}{R_{5}} s \nu\left(N p^{2}+2 p q\right)\right] \exp \left[\frac{s}{R_{5}}(N p+q)\left(x^{4}+i s x^{5}\right)\right] .
\end{aligned}
$$

Each $C_{q}^{s}$ is determined by the normalization condition, i.e.,

$$
\int_{0}^{2 \pi R_{4}} d x^{4} \int_{0}^{2 \pi R_{5}} d x^{5} \psi_{\bar{q}}^{s, *} \psi_{q}^{s}=\delta_{\bar{q} q}
$$

From eq. (3.9),

$$
\left|C_{q}^{s}\right|^{2} \cdot 2 \pi R_{5} \exp \left[\frac{2 \pi R_{4}}{N R_{5}} q^{2}\right] \sqrt{\frac{\pi}{|F|}}=1
$$

Eventually,

$$
\begin{aligned}
\psi_{q}^{s}\left(x^{4}, x^{5}\right)= & \left(2 \pi R_{5} \sqrt{\frac{\pi}{|F|}}\right)^{-1 / 2} \exp \left[-\frac{|F|}{2}\left(x^{4}\right)^{2}\right] \\
& \times \sum_{p \in \mathbb{Z}} \exp \left[-\frac{\pi R_{4}}{R_{5}} s \nu\left(N p^{2}+2 p q\right)\right] \exp \left[\frac{s}{R_{5}}(N p+q)\left(x^{4}+i s x^{5}\right)\right] \\
= & \left(2 \pi R_{5} \sqrt{\frac{\pi}{|F|}}\right)^{-1 / 2} \exp \left[-\frac{|F|}{2}\left(x^{4}\right)^{2}\right] \vartheta\left[\begin{array}{c}
q / N \\
0
\end{array}\right]\left(\frac{s N}{2 \pi i R_{5}}\left(x^{4}+i s x^{5}\right), i \frac{R_{4}}{R_{5}} N\right),
\end{aligned}
$$

where the function $\vartheta$ is the Jacobi theta-function

$$
\vartheta\left[\begin{array}{l}
a \\
b
\end{array}\right](\nu, \tau)=\sum_{p \in \mathbb{Z}} \exp \left[\pi i(a+p)^{2} \tau+2 \pi i(a+p)(\nu+b)\right],
$$

where $\operatorname{Im} \tau>0$.

We can easily generalize the above discussion to non-Abelian, bi-fundamental or higher dimensional torus. 


\section{Dirac operator on the NC torus and the IKKT model}

In this section, we introduce differential operators on the $\mathrm{NC}$ torus and construct them from the IKKT model.

\subsection{Differential operators on the NC torus}

Let $\mathcal{A}$ be a $C^{*}$-algebra over $\mathbb{C}$ and $d: \mathcal{A} \rightarrow \mathcal{A}$ be a linear map. $d$ is called a derivation on $\mathcal{A}$ if $d$ satisfies the Leibniz rule, i.e.,

$$
d(a b)=(d a) b+a(d b), \quad d(\lambda a)=\lambda(d a)
$$

for all $a, b \in \mathcal{A}, \lambda \in \mathbb{C}$.

In mathematics, the NC torus is the $C^{*}$-algebra generated by $\hat{U}_{4}$ and $\hat{U}_{2}$ satisfying eq. (2.12). Therefore, derivations are completely defined by how they act on the two generators. We define

$$
\delta^{4} \hat{U}_{4}=\frac{i}{R_{4}} \hat{U}_{4}, \quad \delta^{5} \hat{U}_{5}=\frac{i}{R_{5}} \hat{U}_{5}, \quad \delta^{4} \hat{U}_{5}=\delta^{5} \hat{U}_{4}=0
$$

and satisfy the linearity and the Leibniz rule. $\delta^{i}(i=4,5)$ is called the basic derivation. The factors $\frac{1}{R_{4}}$ and $\frac{1}{R_{5}}$ come from the definition of the torus, cf., subsection 3.1. We can confirm that the basic derivations commute each other.

The Dirac operator on the NC torus is defined by the basic derivations

$$
\not D:=i \sum_{i=4,5} \sigma_{i} \delta^{i}
$$

where $\sigma_{4}$ and $\sigma_{5}$ are the Pauli matrices $\sigma_{1}$ and $\sigma_{2}$, respectively.

\subsection{Dirac operator on the NC torus based on the IKKT model}

In this subsection, we construct the differential operators, which are introduced in the previous subsection, based on the IKKT model. First, we focus on noncommutative SYM theory based on the IKKT model [15].

We consider the expansion of the action (2.1) around the specific background: spacetime $\hat{X}_{\mathrm{bg}}^{M}$ satisfying eq. $(2.8)$, gauge field $\hat{A}_{\mathrm{bg}, M}$ will be defined in section 5 . Namely,

$$
\hat{X}^{M}=\hat{X}_{\mathrm{bg}}^{M}+\theta^{M N}\left(\hat{A}_{\mathrm{bg}, N}+\hat{A}_{N}\right) .
$$

We refer to appendix A for the details. A point is that the partial derivatives are defined as

$$
\partial_{M}:=-i\left(\theta^{-1}\right)_{M N}\left[\hat{X}_{\mathrm{bg}}^{N}, \cdot\right],
$$

where $\left(\theta^{-1}\right)_{M N}$ is the inverse matrix of $\theta^{M N}$. We can confirm that the partial derivatives satisfy $\partial_{M} \hat{X}_{\mathrm{bg}}^{N}=\delta_{M}^{N}$. Here, we assume that $\theta^{M N}$ is non-degenerate. In the following, we identify $\hat{X}_{\text {bg }}^{M}$ with eq. (2.14) (i.e., we mainly focus on the NC torus $(M=0 \sim 9 \rightarrow i=4,5)$ ), and we omit the subscription "bg". 
The action of $\partial_{i}$ on the algebra of the NC torus is

$$
\begin{aligned}
\partial_{i} \hat{U}_{j} & =-i\left(\theta^{-1}\right)_{i k}\left[\hat{X}^{k}, \hat{U}_{j}\right] \\
& =\left(\theta^{-1}\right)_{i j} \cdot 2 \pi i R_{j} \hat{U}_{j} .
\end{aligned}
$$

Therefore, the basic derivations on the NC torus based on the IKKT model are defined as

$$
\delta^{i}:=\frac{1}{2 \pi\left(R_{i}\right)^{2}} \theta^{i j} \partial_{j}
$$

We can confirm that the basic derivations (4.4) satisfy eq. (4.1). In addition, eq. (2.8) and the Jacobi identity assures the commutativity of the basic derivations (4.4).

From the above, we propose a Dirac operator on the NC torus that is consistent with the IKKT model as follows, ${ }^{4}$

$$
\not D_{\mathrm{phys}}:=2 \pi i \sum_{i=4,5}\left(R_{i}\right)^{2} \sigma_{i} \delta^{i}
$$

In the fermionic part of the action (2.1),

$$
\not D_{6}=\sum_{i=4}^{9} \Gamma^{i}\left[\hat{X}_{i}, \cdot\right]
$$

is identified as a Dirac operator on the extra dimensions, e.g., [17]. As mentioned above, we focus on the first two extra dimensions that are the NC torus (i.e., $\sum_{i=4}^{9} \rightarrow \sum_{i=4,5}$ ). We can verify that eqs. (4.5) and (4.6) are equivalent. Namely,

$$
\begin{aligned}
\not_{\mathrm{phys}} \hat{\psi} & =2 \pi i \sum_{i=4,5}\left(R_{i}\right)^{2} \sigma_{i} \delta^{i} \hat{\psi} \\
& =i \sigma_{i} \theta^{i j}\left(-i\left(\theta^{-1}\right)_{j k}\left[\hat{X}^{k}, \hat{\psi}\right]\right) \\
& =\sigma_{i}\left[\hat{X}^{i}, \hat{\psi}\right] \\
& =\not D_{6} \hat{\psi}
\end{aligned}
$$

Therefore, the operator (4.5) is a suitable Dirac operator on the NC torus based on the IKKT model.

The partial derivatives are defined by eq. (4.3), and the gauge field is introduced by eq. (4.2). The covariant derivatives are naturally defined by

$$
\partial_{M} \rightarrow D_{M}:=-i\left(\theta^{-1}\right)_{M N}\left[\hat{X}_{\mathrm{bg}}^{N}+\theta^{N L} \hat{A}_{L}, \cdot\right]=\partial_{M}-i\left[\hat{A}_{M}, \cdot\right] .
$$

However, we cannot introduce any fermions $\hat{\psi}$ in a fundamental representation, i.e., $\hat{\psi} \rightarrow$ $\hat{\Omega} \hat{\psi}$, where $\hat{\Omega}$ is a unitary operator as a gauge transformation. This comes from eq. (2.10).

\footnotetext{
${ }^{4}$ The subscription "phys" means that the Dirac operator (4.5) has the mass dimension 1. This mass dimension is the same with the usual Dirac operator in QFT.
} 
On the other hand, we can introduce fermions in a fundamental representation if we define the covariant derivatives as

$$
D_{M}:=\partial_{M}-i \hat{A}_{M}
$$

However, the original action (2.1) does not have the field strength which is defined by the covariant derivatives (4.8). Therefore, if we want to consider fermions in a fundamental representation, we should realize as a part of the action (2.1). Let us consider, for example, $\mathrm{U}(2)$ gauge theory with an adjoint matter based on the action (2.1), i.e.,

$$
\hat{X}_{\mathrm{U}(2)}^{M}:=\hat{X}_{\mathrm{U}(1)}^{M} \times 1_{2 \times 2}, \quad \hat{A}_{M}=\left(\begin{array}{cc}
\hat{A}_{M}^{11} & \hat{A}_{M}^{12} \\
\hat{A}_{M}^{21} & \hat{A}_{M}^{22}
\end{array}\right), \quad \hat{\psi}=\left(\begin{array}{cc}
\hat{\psi}^{11} & \hat{\psi}^{12} \\
\hat{\psi}^{21} & \hat{\psi}^{22}
\end{array}\right),
$$

where $\hat{X}_{(\mathrm{U}(1)}^{M}$ satisfies $\left[\hat{X}_{(\mathrm{U}(1)}^{M}, \hat{X}_{(\mathrm{U}(1)}^{N}\right]=i \theta^{M N}$. We assume that the gauge group breaks such that $\mathrm{U}(2) \rightarrow \mathrm{U}(1) \times \mathrm{U}(1) \rightarrow \mathrm{U}(1)$ : top left component of $\hat{A}_{i}$. If we focus on only $\hat{\psi}^{12}$ and ignore other components of $\hat{A}_{i}$, then we can derive the action with the fermion in $\mathrm{U}(1)$ fundamental representation.

\section{Zero-mode analysis on the magnetized NC torus}

\subsection{Twisted bundle on the NC torus}

To consider the twisted bundle on the NC torus, we introduce a background gauge field

$$
\hat{A}_{4}\left(\hat{X}^{4}, \hat{X}^{5}\right)=0, \quad \hat{A}_{5}\left(\hat{X}^{4}, \hat{X}^{5}\right)=\mathcal{F} \hat{X}^{4}
$$

and the field strength is $\hat{F}_{45}=\mathcal{F}$.

The gauge transformation is obtained by

$$
\hat{A}_{M} \rightarrow \hat{A}_{M}^{\prime}=\hat{\Omega} \hat{A}_{M} \hat{\Omega}^{-1}+i \hat{\Omega} \partial_{M} \hat{\Omega}^{-1}
$$

where $\hat{\Omega}$ is a unitary operator.

The background gauge field is varied by the torus translations (2.11), i.e.,

$$
\begin{aligned}
& \hat{A}_{4}\left(\hat{X}^{4}+2 \pi R_{4}, \hat{X}^{5}\right)=\hat{A}_{4}\left(\hat{X}^{4}, \hat{X}^{5}+2 \pi R_{5}\right)=0 \\
& \hat{A}_{5}\left(\hat{X}^{4}+2 \pi R_{4}, \hat{X}^{5}\right)=\hat{A}_{5}\left(\hat{X}^{4}, \hat{X}^{5}\right)+2 \pi R_{4} \mathcal{F} \\
& \hat{A}_{5}\left(\hat{X}^{4}, \hat{X}^{5}+2 \pi R_{5}\right)=\hat{A}_{5}\left(\hat{X}^{4}, \hat{X}^{5}\right) .
\end{aligned}
$$

We can realize eq. (5.2) as the gauge transformations, i.e.,

$$
\hat{\Omega}_{4}\left(\hat{X}^{4}, \hat{X}^{5}\right) \propto \exp \left[\frac{2 \pi i R_{4}}{1+\theta^{45} \mathcal{F}} \cdot \mathcal{F} \hat{X}^{5}\right], \quad \hat{\Omega}_{5}\left(\hat{X}^{4}, \hat{X}^{5}\right) \propto \hat{1},
$$

where $\propto$ represents an action on $\mathbb{C}^{m}$ part. This part does not affect eq. (5.2) since the $\mathbb{C}^{m}$ part of $\hat{X}^{4}$ and $\hat{X}^{5}$ is the identity matrix. Therefore, in the following, we assume this part is the identity matrix and omit. 
Next, we must consider the consistency condition corresponding to eq. (3.3). Namely,

$$
\hat{\Omega}_{5}\left(\hat{X}^{4}+2 \pi R_{4}, \hat{X}^{5}\right) \hat{\Omega}_{4}\left(\hat{X}^{4}, \hat{X}^{5}\right)=\hat{\Omega}_{4}\left(\hat{X}^{4}, \hat{X}^{5}+2 \pi R_{5}\right) \hat{\Omega}_{5}\left(\hat{X}^{4}, \hat{X}^{5}\right) .
$$

Eq. (5.4) implies that the magnetic flux $\mathcal{F}$ is quantized such that

$$
\frac{\mathcal{F}}{1+\theta^{45 \mathcal{F}}} \cdot \frac{A}{2 \pi}=\mathcal{N} \in \mathbb{Z}
$$

\subsection{Zero-modes of the Dirac operator $\not D_{\text {phys }}$}

In this subsection, we consider the zero-mode equations of the Dirac operator $\not D_{\text {phys }}$ with the background gauge field (5.1). In the following, we show the zero-mode solutions in (i) fundamental representation (ii) bifundamental representation.

Case (i). In this case, the zero-mode equation is

$$
\not D_{\text {phys }} \hat{\psi}=\theta^{45}\left(\begin{array}{cc}
0 & -\left(\partial_{4}-i \partial_{5}-\mathcal{F} \hat{X}^{4}\right) \\
\partial_{4}+i \partial_{5}+\mathcal{F} \hat{X}^{4} & 0
\end{array}\right)\left(\begin{array}{l}
\hat{\psi}^{+} \\
\hat{\psi}^{-}
\end{array}\right)=0,
$$

where $\hat{\psi}^{s}(s= \pm 1)$ is in the fundamental representation. Simply,

$$
\left(\partial_{4}+i s \partial_{5}+s \mathcal{F} \hat{X}^{4}\right) \hat{\psi}^{s}=0 \quad(s= \pm 1)
$$

with the periodic boundary conditions

$$
\begin{aligned}
& \hat{\psi}^{s}\left(\hat{X}^{4}+2 \pi R_{4}, \hat{X}^{5}\right)=\exp \left[\frac{2 \pi i R_{4}}{1+\theta^{45} \mathcal{F}} \cdot \mathcal{F} \hat{X}^{5}\right] \hat{\psi}^{s}\left(\hat{X}^{4}, \hat{X}^{5}\right) \\
& \hat{\psi}^{s}\left(\hat{X}^{4}, \hat{X}^{5}+2 \pi R_{5}\right)=\hat{\psi}^{s}\left(\hat{X}^{4}, \hat{X}^{5}\right)
\end{aligned}
$$

Although eq. (5.6) is written by the operators, this equation has the same form with eq. (3.6). Therefore, we expect that the zero-mode equation (5.6) can be constructed from that of eq. (3.6). Here, we consider the Fourier transformations. The Fourier transformation of the whole zero-mode solution of eq. (3.6) is obtained by

$$
\psi^{s}\left(x^{4}, x^{5}\right)=\int \frac{d k}{2 \pi} \sum_{n=|N| p+q \in \mathbb{Z}} C \exp \left[-\frac{k^{2}}{2|F|}-\frac{i k n}{R_{5} F}\right] \exp \left[i k x^{4}\right] \exp \left[i \frac{n}{R_{5}} x^{5}\right],
$$

where $C$ is the normalization constant and the magnetic flux $F$ satisfies the quantization condition (3.4). We expect that the whole solution of eq. (5.6) is obtained by

$$
\hat{\psi}^{s}\left(\hat{X}^{4}, \hat{X}^{5}\right)=\int \frac{d k}{2 \pi} \sum_{n=|N| p+q \in \mathbb{Z}} C \exp \left[-\frac{k^{2}}{2|F|}-\frac{i k n}{R_{5} F}\right] \exp \left[i k \hat{X}^{4}\right] \exp \left[i \frac{n}{R_{5}} \hat{X}^{5}\right] .
$$

However, eq. (5.8) does not satisfy eq. (5.6) because the quantization conditions for the magnetic flux are different.

Here, we focus on that $p$ and $q$ do not appear alone in eq. (5.7). This fact allows us to vary the decomposition of the label from $n=|N| p+q$ to $n=|\mathcal{N}| p+q$. In addition, 
we should replace the magnetic flux $F$ with $\mathcal{F}$ satisfying (5.5). Then, we can obtain the zero-mode solutions, ${ }^{5}$ i.e.,

$$
\hat{\psi}^{s}\left(\hat{X}^{4}, \hat{X}^{5}\right)=\int \frac{d k}{2 \pi} \sum_{n=|\mathcal{N}| p+q \in \mathbb{Z}} C \exp \left[-\frac{k^{2}}{2|\mathcal{F}|}-\frac{i k n}{R_{5} \mathcal{F}}\right] \exp \left[i k \hat{X}^{4}\right] \exp \left[i \frac{n}{R_{5}} \hat{X}^{5}\right],
$$

or

$$
\hat{\psi}_{q}^{s}\left(\hat{X}^{4}, \hat{X}^{5}\right)=\int \frac{d k}{2 \pi} \sum_{p \in \mathbb{Z}} C \exp \left[-\frac{k^{2}}{2|\mathcal{F}|}-\frac{i k}{R_{5} \mathcal{F}}(|\mathcal{N}| p+q)\right] \exp \left[i k \hat{X}^{4}\right] \exp \left[i \frac{|\mathcal{N}| p+q}{R_{5}} \hat{X}^{4}\right],
$$

where $q=0 \sim|\mathcal{N}|-1$. We can confirm that the above expansions satisfy eq. (5.6) if $\mathcal{F} s>0$. The normalization constant $C$ will be computed in subsection 5.4.

However, this form is difficult to use when we compute the normalization constant and Yukawa couplings. Fortunately, we can rewrite by using the Jacobi theta-function

$$
\hat{\psi}_{q}^{s}\left(\hat{X}^{4}, \hat{X}^{5}\right)=C \sqrt{\frac{|\mathcal{F}|}{2 \pi}} \exp \left[-\frac{|\mathcal{F}|}{2}\left(\hat{X}^{4}\right)^{2}\right] \vartheta\left[\begin{array}{c}
q / \mathcal{N} \\
0
\end{array}\right]\left(\frac{s|\mathcal{N}|}{2 \pi i R_{5}}\left(\hat{X}^{4}+i s \hat{X}^{5}\right), i \frac{R_{4}}{R_{5}} s \mathcal{N}\right) .
$$

If we require $s \mathcal{N}>0$, eq. (5.10) can be interpreted as the operator form of eq. (3.11) up to the normalization constant. Therefore, in the following, we restrict ourselves to the case $s \mathcal{N}>0$.

Case(ii). For simplicity, we consider the magnetic flux breaks the gauge group $\mathrm{U}(2) \rightarrow$ $\mathrm{U}(1) \times \mathrm{U}(1)$, i.e.,

$$
\hat{A}_{4}\left(\hat{X}^{4}, \hat{X}^{5}\right)=0, \quad \hat{A}_{5}\left(\hat{X}^{4}, \hat{X}^{5}\right)=\left(\begin{array}{cc}
\mathcal{F}_{1} \hat{X}^{4} & 0 \\
0 & \mathcal{F}_{2} \hat{X}^{5}
\end{array}\right)
$$

where $\mathcal{F}_{i}(i=1,2)$ satisfies (5.5).

In this case, we consider a fermion in $\mathrm{U}(2)$ adjoint representation,

$$
\hat{\psi}=\left(\begin{array}{c}
\hat{\psi}^{+} \\
\hat{\psi}^{-}
\end{array}\right), \quad \hat{\psi}^{s}=\left(\begin{array}{cc}
\hat{\psi}_{11}^{s} & \hat{\psi}_{12}^{s} \\
\hat{\psi}_{21}^{s} & \hat{\psi}_{22}^{s}
\end{array}\right) .
$$

The zero-mode equation for each chirality is written as

$$
\left(\partial_{4}+i s \partial_{5}\right)\left(\begin{array}{cc}
\hat{\psi}_{11}^{s} & \hat{\psi}_{12}^{s} \\
\hat{\psi}_{21}^{s} & \hat{\psi}_{22}^{s}
\end{array}\right)+s\left(\begin{array}{cc}
\mathcal{F}_{1}\left[\hat{X}^{4}, \hat{\psi}_{11}^{s}\right] & \mathcal{F}_{1} \hat{X}^{4} \hat{\psi}_{12}^{s}-\mathcal{F}_{2} \hat{\psi}_{12}^{s} \hat{X}^{4} \\
\mathcal{F}_{2} \hat{X}^{4} \hat{\psi}_{21}^{s}-\mathcal{F}_{1} \hat{\psi}_{21}^{s} \hat{X}^{4} & \mathcal{F}_{2}\left[\hat{X}^{4}, \hat{\psi}_{22}^{s}\right]
\end{array}\right)=0 \quad(s= \pm 1) .
$$

First, we consider the top right component $\hat{\psi}_{12}^{s}$. Precisely, the zero-mode equation of $\hat{\psi}_{12}^{s}$ is

$$
\left(\partial_{4}+i s \partial_{5}\right) \hat{\psi}_{12}^{s}+s\left(\mathcal{F}_{1} \hat{X}^{4} \hat{\psi}_{12}^{s}-\mathcal{F}_{2} \hat{\psi}_{12}^{s} \hat{X}^{4}\right)=0
$$

\footnotetext{
${ }^{5}$ We can obtain the same result by using the Baker-Campbell-Hausdorff formula.
} 
with the periodic boundary conditions

$$
\begin{aligned}
& \hat{\psi}_{12}^{s}\left(\hat{X}^{4}+2 \pi R_{4}, \hat{X}^{5}\right)=\exp \left[\frac{2 \pi i R_{4}}{1+\theta^{45} \mathcal{F}_{1}} \cdot \mathcal{F}_{1} \hat{X}^{5}\right] \hat{\psi}_{12}^{s}\left(\hat{X}^{4}, \hat{X}^{5}\right) \exp \left[-\frac{2 \pi i R_{4}}{1+\theta^{45} \mathcal{F}_{2}} \cdot \mathcal{F}_{2} \hat{X}^{5}\right] \\
& \hat{\psi}_{12}^{s}\left(\hat{X}^{4}, \hat{X}^{5}+2 \pi R_{5}\right)=\hat{\psi}_{12}^{s}\left(\hat{X}^{4}, \hat{X}^{5}\right)
\end{aligned}
$$

In the case (i), $\exp \left[-\frac{|\mathcal{F}|}{2}\left(\hat{X}^{4}\right)^{2}\right]$ of eq. (5.10) corresponds to the term: $s \mathcal{F} \hat{X}^{4}$ in eq. (5.6). From this observation, we can obtain the solutions of eq. (5.11) as

$$
\begin{aligned}
\hat{\psi}_{12}^{s}\left(\hat{X}^{4}, \hat{X}^{5}\right)= & \int \frac{d k}{2 \pi} \sum_{n=\left|\mathcal{N}_{12}\right| p+q \in \mathbb{Z}} C_{12} \exp \left[-\frac{k^{2}}{2\left|\mathcal{F}_{12}\right|}-\frac{i k n}{R_{5} \mathcal{F}_{12}}\left(1+\theta^{12} \mathcal{F}_{2}\right)\right] \\
& \times \exp \left[i k \hat{X}^{4}\right] \exp \left[i \frac{n}{R_{5}} \hat{X}^{5}\right]
\end{aligned}
$$

or

$$
\begin{aligned}
\hat{\psi}_{12, q}^{s}\left(\hat{X}^{1}, \hat{X}^{2}\right)= & C_{12} \sqrt{\frac{\left|\mathcal{F}_{12}\right|}{2 \pi}} \exp \left[-\frac{s_{12} \mathcal{F}_{1}}{2}\left(\hat{X}^{1}\right)^{2}\right] \\
& \times \vartheta\left[\begin{array}{c}
q /\left|\mathcal{N}_{12}\right| \\
0
\end{array}\right]\left(\frac{s\left|\mathcal{N}_{12}\right|}{2 \pi i R_{5}}\left(\hat{X}^{1}+i s \hat{X}^{2}\right), i \frac{R_{4}}{R_{5}}\left|\mathcal{N}_{12}\right|\right) \exp \left[\frac{s_{12} \mathcal{F}_{2}}{2}\left(\hat{X}^{1}\right)^{2}\right]
\end{aligned}
$$

where $\mathcal{F}_{12}=\mathcal{F}_{1}-\mathcal{F}_{2}, s_{12}=\operatorname{sign}\left(\mathcal{F}_{12}\right), \mathcal{N}_{12}=\mathcal{N}_{1}-\mathcal{N}_{2}$, and we restrict ourselves to $s_{12} \mathcal{N}_{12}$. We can confirm that eq. (5.12) satisfies eq. (5.11) and the periodic boundary conditions if $s_{12} \mathcal{F}_{12}>0$.

Next, we consider the top left component $\hat{\psi}_{11}^{s}$. The zero-mode equation of $\hat{\psi}_{11}^{s}$ is written as

$$
\left(\partial_{4}+i s \partial_{5}\right) \hat{\psi}_{11}^{s}+s \mathcal{F}_{1}\left[\hat{X}^{4}, \hat{\psi}_{11}^{s}\right]=0
$$

with the periodic boundary conditions

$$
\begin{aligned}
& \hat{\psi}_{11}^{s}\left(\hat{X}^{4}+2 \pi R_{4}, \hat{X}^{5}\right)=\exp \left[\frac{2 \pi i R_{4}}{1+\theta^{45} \mathcal{F}_{1}} \cdot \mathcal{F}_{1} \hat{X}^{5}\right] \hat{\psi}_{11}^{s}\left(\hat{X}^{4}, \hat{X}^{5}\right) \exp \left[-\frac{2 \pi i R_{4}}{1+\theta^{45} \mathcal{F}_{1}} \cdot \mathcal{F}_{1} \hat{X}^{5}\right], \\
& \hat{\psi}_{11}^{s}\left(\hat{X}^{4}, \hat{X}^{5}+2 \pi R_{5}\right)=\hat{\psi}_{11}^{s}\left(\hat{X}^{4}, \hat{X}^{5}\right) .
\end{aligned}
$$

In this case, the right-hand side of the first condition of (5.13) corresponds to the shift of $\hat{X}^{4}$ such that $\hat{X}^{4} \rightarrow \hat{X}^{4}+\frac{\mathcal{N}_{1} \theta^{45}}{R_{5}}$. This shift implies $\frac{\mathcal{F}_{1} \theta^{45}}{1+\theta^{45} \mathcal{F}_{1}}=1$, and this is a contradiction. Therefore, $\hat{\psi}_{11}^{s}$ must be the scalar operator, i.e., $\hat{\psi}_{11}^{s}=$ const. $\times \mathbf{1}$.

Obviously, the above results can be generalized to the magnetic fluxes break the gauge group $\mathrm{U}(N) \rightarrow \prod_{a=1}^{n} \mathrm{U}\left(N_{a}\right)$, where $\sum_{a=1}^{n} N_{a}=N$.

\subsection{Eigenvalues of the Laplacian}

In the IKKT model, $\square:=\sum_{i}\left[\hat{X}^{i},\left[\hat{X}^{i}, \cdot\right]\right]$ (in this paper, $\square:=\sum_{i=4,5}\left[\hat{X}^{i},\left[\hat{X}^{i}, \cdot\right]\right]$ ) is identified as the Laplacian (e.g., [2,3] or eq. (A.6)). The eigenvalue problem of the Laplacian relates to that of the square of the Dirac operator. Let us rewrite the Dirac operator as

$$
\not D_{\mathrm{phys}}=\left(\begin{array}{cc}
0 & -D \\
D^{\prime} & 0
\end{array}\right),
$$


then

$$
\not D_{\mathrm{phys}}^{2}=\left(\begin{array}{cc}
-D D^{\prime} & 0 \\
0-D^{\prime} D
\end{array}\right)=\square+\left(\begin{array}{cc}
-\frac{\left(\theta^{45}\right)^{2}}{2}\left[D, D^{\prime}\right] & 0 \\
0 & -\frac{\left(\theta^{45}\right)^{2}}{2}\left[D^{\prime}, D\right]
\end{array}\right) .
$$

The action of $(5.14)$ on $(1,2)$ component of the fermion is written as

$$
\not D_{\text {phys }}^{2} \hat{\psi}_{12}^{s}=\square \hat{\psi}_{12}^{s}-s\left(\theta^{45}\right)^{2} \mathcal{F}_{12} \hat{\psi}_{12}^{s} .
$$

From the above, $D^{\prime}(D)$ has the zero-mode solutions if $\mathcal{F}_{12}>0\left(\mathcal{F}_{12}<0\right)$. Therefore, we can see that eq. (5.12) is not only the zero-modes of the Dirac operator but also the lightest mode of the Laplacian.

In addition, we can construct the eigenmodes of the Laplacian corresponding $(1,2)$ component. We focus on the commutation relation of $D$ and $D^{\prime}$,

$$
\left[D, D^{\prime}\right] \hat{\psi}_{12}^{s}=2\left(\theta^{45}\right)^{2} \mathcal{F}_{12} \hat{\psi}_{12}^{s} .
$$

If we select $\mathcal{F}_{12}>0, D$ and $-D^{\prime}$ can play roles of the creation operator and the annihilation operator, respectively. By considering the analogy of the harmonic oscillator, i.e.,

$$
N:=-D D^{\prime}, \quad \square=N+\left(\theta^{45}\right)^{2} \mathcal{F}_{12},
$$

then the eigenmodes of the Laplacian are obtained by

$$
\begin{aligned}
\square \hat{\psi}_{12}^{+, n} & =\lambda_{n} \hat{\psi}_{12}^{+, n}, \\
\hat{\psi}_{12}^{+, n} & :=D^{n} \hat{\psi}_{12}^{+}, \quad \lambda_{n}=\left(\theta^{45}\right)^{2} \mathcal{F}_{12}(2 n+1) .
\end{aligned}
$$

If we select $\mathcal{F}_{12}<0$, it is sufficient to reverse the roles of $D$ and $D^{\prime}$.

In the commutative case, ${ }^{6}$ the spectrum of the Laplacian is obtained by $\lambda_{n}=F_{12}(2 n+$ 1 ), where $F_{i}(i=1,2)$ is the magnetic flux satisfying (3.4) (cf., [7]) and $F_{12}=F_{1}-F_{2}$. It seems that the spectrum vanishes in the limit $\theta^{45} \rightarrow 0$. However, the bosonic part of the effective action is the fourth order for the $\mathrm{NC}$ parameter if we ignore the order included in the definition of the partial derivatives. Therefore, $\left(\theta^{45}\right)^{2}$ of the spectrum is necessary, but the limit $\theta^{45} \rightarrow 0$ is non-trivial.

\subsection{Normalizations and Yukawa couplings}

Normalizations. Let us start from the normalization constant of eq. (5.12) since $\mathcal{F}_{2}=0$ corresponds to the case (i).

$$
\begin{aligned}
1=\operatorname{Tr}\left(\hat{\psi}_{12, q}^{s, \dagger}\left(\hat{X}^{4}, \hat{X}^{5}\right) \hat{\psi}_{12, q^{\prime}}^{s}\left(\hat{X}^{4}, \hat{X}^{5}\right)\right) & :=\int_{0}^{2 \pi R_{5}} d X^{5}\left\langle X^{5}\left|\hat{\psi}_{12, q}^{s, \dagger}\left(\hat{X}^{4}, \hat{X}^{5}\right) \hat{\psi}_{12, q^{\prime}}^{s}\left(\hat{X}^{4}, \hat{X}^{5}\right)\right| X^{5}\right\rangle \\
& =\frac{1}{A} \int_{0}^{2 \pi R_{4}} d X^{4} \int_{0}^{2 \pi R_{5}} d X^{5} \hat{\psi}_{12, q}^{s, \dagger}\left(X^{4}, X^{5}\right) \hat{\psi}_{12, q^{\prime}}^{s}\left(X^{4}, X^{5}\right) \\
& =\delta_{q q^{\prime}}\left|C_{12}\right|^{2} \frac{R_{5}\left|\mathcal{F}_{12}\right|}{A} \cdot I_{12}\left(\theta^{12}\right),
\end{aligned}
$$

\footnotetext{
${ }^{6}$ We use commutative in the sense of usual torus $T^{2}$, not the case: $\left[X^{M}, X^{N}\right]=0$ (for all $M, N=0 \sim 9$ ).
} 
where

$$
I_{12}\left(\theta^{12}\right):=\sum_{p \in \mathbb{Z}} \int_{\frac{p\left|\mathcal{N}_{12}\right| p+q}{R_{5} \mathcal{F}_{12}}\left(1+\theta^{45} \mathcal{F}_{2}\right)}^{\frac{p\left|\mathcal{N}_{12}\right| p+q}{R_{5} \mathcal{F}_{12}}\left(1+\theta^{45} \mathcal{F}_{2}\right)-2 \pi R_{4}} \exp \left[-\left|\mathcal{F}_{12}\right| x^{2}\right] d x
$$

is a function satisfies $I_{12}(0)=\sqrt{\pi /\left|\mathcal{F}_{12}\right|}$ (the Gaussian integral). Therefore, the normalization constant $C_{12}$ is

$$
C_{12}=\left(\frac{R_{5}\left|\mathcal{F}_{12}\right|}{A} \cdot I_{12}\left(\theta^{12}\right)\right)^{-1 / 2}
$$

We should note the definition of the trace on the infinite dimensional space. For example,

$$
\begin{aligned}
\int_{0}^{2 \pi R_{4}} d X^{4}\left\langle X^{4}\left|\hat{f}\left(\hat{X}^{4}, \hat{X}^{5}\right)\right| X^{4}\right\rangle & =\int_{0}^{2 \pi R_{4}} d X^{4} \int_{0}^{2 \pi R_{5}} d X^{5}\left\langle X^{4}\left|\hat{f}\left(\hat{X}^{4}, \hat{X}^{5}\right)\right| X^{5}\right\rangle\left\langle X^{5} \mid X^{4}\right\rangle \\
& =\int_{0}^{2 \pi R_{4}} d X^{4} \int_{0}^{2 \pi R_{5}} d X^{5}\left\langle X^{5} \mid X^{4}\right\rangle\left\langle X^{4}\left|\hat{f}\left(\hat{X}^{4}, \hat{X}^{5}\right)\right| X^{5}\right\rangle \\
& =\int_{0}^{2 \pi R_{5}} d X^{5}\left\langle X^{5}\left|\hat{f}\left(\hat{X}^{4}, \hat{X}^{5}\right)\right| X^{5}\right\rangle \\
& =\operatorname{Tr}\left(\hat{f}\left(\hat{X}^{4}, \hat{X}^{5}\right)\right) .
\end{aligned}
$$

In addition, we should confirm that the trace is well-defined with respect to the compactification conditions (2.11). In this paper, target operators $\hat{f}$ are periodic with respect to the $X^{5}$-direction. This means

$$
\begin{aligned}
\int_{2 \pi R_{5}}^{4 \pi R_{5}} d X^{5}\left\langle X^{5}\left|\hat{f}\left(\hat{X}^{4}, \hat{X}^{5}\right)\right| X^{5}\right\rangle & =\int_{0}^{2 \pi R_{5}} d X^{5}\left\langle X^{5}+2 \pi R_{5}\left|\hat{f}\left(\hat{X}^{4}, \hat{X}^{5}\right)\right| X^{5}+2 \pi R_{5}\right\rangle \\
& =\int_{0}^{2 \pi R_{5}} d X^{5}\left\langle X^{5}\left|\hat{U}_{5} \hat{f}\left(\hat{X}^{4}, \hat{X}^{5}\right) \hat{U}_{5}^{-1}\right| X^{5}\right\rangle \\
& =\int_{0}^{2 \pi R_{5}} d X^{5}\left\langle X^{5}\left|\hat{f}\left(\hat{X}^{4}, \hat{X}^{5}+2 \pi R_{5}\right)\right| X^{5}\right\rangle \\
& =\int_{0}^{2 \pi R_{5}} d X^{5}\left\langle X^{5}\left|\hat{f}\left(\hat{X}^{4}, \hat{X}^{5}\right)\right| X^{5}\right\rangle \\
& =\operatorname{Tr}\left(\hat{f}\left(\hat{X}^{4}, \hat{X}^{5}\right)\right) .
\end{aligned}
$$

On the other hand, the target operators $\hat{f}$ are quasiperiodic with respect to the $X^{4}$ direction. Since the unitary operator for the quasiperiodicity $\hat{\Omega}_{4}$ is written by $\hat{X}^{5}\left(\hat{\Omega}_{4}\right.$ 
is also periodic with respect to the $X^{5}$-direction), then

$$
\begin{aligned}
\int_{2 \pi R_{4}}^{4 \pi R_{4}} d X^{4}\left\langle X^{4}\left|\hat{f}\left(\hat{X}^{4}, \hat{X}^{5}\right)\right| X^{4}\right\rangle & =\int_{0}^{2 \pi R_{4}} d X^{4}\left\langle X^{4}+2 \pi R_{4}\left|\hat{f}\left(\hat{X}^{4}, \hat{X}^{5}\right)\right| X^{4}+2 \pi R_{4}\right\rangle \\
& =\int_{0}^{2 \pi R_{4}} d X^{4}\left\langle X^{4}\left|\hat{U}_{4} \hat{f}\left(\hat{X}^{4}, \hat{X}^{3}\right) \hat{U}_{4}^{-1}\right| X^{4}\right\rangle \\
& =\int_{0}^{2 \pi R_{4}} d X^{4}\left\langle X^{4}\left|\hat{\Omega}_{4} \hat{f}\left(\hat{X}^{4}, \hat{X}^{5}\right) \hat{\Omega}_{4}^{-1}\right| X^{4}\right\rangle \\
& =\int_{0}^{2 \pi R_{5}} d X^{5}\left\langle X^{5}\left|\hat{\Omega}_{4} \hat{f}\left(\hat{X}^{4}, \hat{X}^{5}\right) \hat{\Omega}_{4}^{-1}\right| X^{5}\right\rangle \\
& =\operatorname{Tr}\left(\hat{f}\left(\hat{X}^{4}, \hat{X}^{5}\right)\right),
\end{aligned}
$$

where we used the equivalence (5.15) in the fourth line.

For general gauge transformations $\hat{U}$, we can show the equivalence between before and after the gauge transformations if we assume the existence of the completeness relation of $\hat{U}$. Therefore, the cyclic property of the trace is held, at least, for the gauge transformations. Therefore, the gauge symmetry of the action (2.1) is still held. Similarly, we can verify that all traces defined by the appropriate completeness relation are equivalent.

Yukawa couplings. In the following, for simplicity, we consider the magnetic fluxes break the gauge group $\mathrm{U}(N) \rightarrow \prod_{a=1}^{3} \mathrm{U}\left(N_{a}\right)$, where $\sum_{a=1}^{3} N_{a}=N$ to compute the Yukawa couplings. The background gauge field is obtained by

$$
\hat{A}_{4}\left(\hat{X}^{5}, \hat{X}^{5}\right)=0, \quad \hat{A}_{5}\left(\hat{X}^{4}, \hat{X}^{5}\right)=\left(\begin{array}{ccc}
\mathcal{F}_{1} \hat{X}^{4} \mathbf{1}_{N_{1}} & 0 & 0 \\
0 & \mathcal{F}_{2} \hat{X}^{4} \mathbf{1}_{N_{2}} & 0 \\
0 & 0 & \mathcal{F}_{3} \hat{X}^{4} \mathbf{1}_{N_{3}}
\end{array}\right),
$$

where $\mathcal{F}_{i}(i=1,2,3)$ satisfies (5.5) and $\mathbf{1}_{N_{i}}$ is the $N_{i} \times N_{i}$ identity matrix.

We should consider a sign assignment of the magnetic fluxes. In the following, we select $\mathcal{F}_{23}, \mathcal{F}_{21}, \mathcal{F}_{13}>0$ (This implies $s_{23}, s_{21}, s_{13}=+1$ and $\mathcal{N}_{23}, \mathcal{N}_{21}, \mathcal{N}_{13}>0$ ). This sign assignment is justified by the relation $\mathcal{F}_{12}+\mathcal{F}_{23}+\mathcal{F}_{31}=0$.

The component of the zero-mode fermions corresponding the above sign assignment is obtained by

$$
\hat{\psi}=\left(\begin{array}{c}
\hat{\psi}^{+} \\
\hat{\psi}^{-}
\end{array}\right), \quad \hat{\psi}^{+}=\left(\begin{array}{ccc}
\text { const. } & 0 & \hat{\psi}_{13, i}^{+} \\
\hat{\psi}_{21, j}^{+} & \text {const. } & \hat{\psi}_{23, k}^{+} \\
0 & 0 & \text { const. }
\end{array}\right), \quad \hat{\psi}^{-}=\hat{\psi}^{+, \dagger},
$$

where $i, j$ and $k$ denote the degeneracies and const. $=\hat{\mathbf{1}}$ (corresponds to the wavefunctions of the gauginos in the commutative case). The lightest mode bosons have the same matrix structure with the (anti-) chiral zero-mode fermions.

Let us denote by $\Phi_{a b, I}(a, b=1,2,3)$ the $(a, b)$ block component of the zero-mode fermions or the lightest mode bosons since their function form is the same. In the action (2.1), the Yukawa couplings are described as the product of three matrices, i.e.,

$$
Y_{I J K}:=\operatorname{Tr}\left(\hat{\Phi}_{23, K}^{\dagger} \cdot \hat{\Phi}_{21, I} \cdot \hat{\Phi}_{13, J}\right) .
$$


First, we focus on the product $\hat{\Phi}_{21, I} \cdot \hat{\Phi}_{13, J}$. If operators commute each other, these operators can be regarded as c-numbers. This allows us to use convenient formulas. From eq. (5.12), this product can be written by

$$
\begin{aligned}
& \hat{\Phi}_{21 . I} \cdot \hat{\Phi}_{13, J} \\
& =\sqrt{\frac{2 \pi\left|\mathcal{F}_{21} \mathcal{F}_{13}\right|}{\left|\mathcal{F}_{23}\right|} \frac{C_{21}}{C_{23}}} \\
& \quad \times \sum_{m \in \mathbb{Z}_{\left|\mathcal{N}_{21}\right|+\left|\mathcal{N}_{13}\right|}} \hat{\Phi}_{23, I+J+\left|\mathcal{N}_{21}\right| m} \times \vartheta\left(\begin{array}{c}
\frac{\left|\mathcal{N}_{13}\right| I-\left|\mathcal{N}_{21}\right| J+\left|\mathcal{N}_{21}\right|\left|\mathcal{N}_{13}\right| m}{\left|\mathcal{N}_{21} \mathcal{N}_{23} \mathcal{N}_{13}\right|} \\
0
\end{array}\right)\left(0, i \frac{R_{4}}{R_{5}}\left|\mathcal{N}_{21} \mathcal{N}_{23} \mathcal{N}_{13}\right|\right),
\end{aligned}
$$

where we used $s_{21}, s_{13}=+1$ and the product formula of the Jacobi theta-function [18]

$$
\begin{aligned}
\vartheta\left[\begin{array}{c}
\frac{r_{1}}{N_{1}} \\
0
\end{array}\right]\left(z_{1}, \tau N_{1}\right) \cdot \vartheta\left[\begin{array}{c}
\frac{r_{2}}{N_{2}} \\
0
\end{array}\right]\left(z_{2}, \tau N_{2}\right) \\
=\sum_{m \in \mathbb{Z}_{N_{1}+N_{2}}\left[\begin{array}{c}
\frac{r_{1}+r_{2}+N_{1} m}{N_{1}+N_{2}} \\
0
\end{array}\right]\left(z_{1}+z_{2}, \tau\left(N_{1}+N_{2}\right)\right)} \\
\quad \times \vartheta\left[\begin{array}{c}
\frac{N_{2} r_{1}-N_{1} r_{2}+N_{1} N_{2} m}{N_{1} N_{2}\left(N_{1}+N_{2}\right)} \\
0
\end{array}\right]\left(z_{1} N_{2}-z_{2} N_{1}, \tau N_{1} N_{2}\left(N_{1}+N_{2}\right)\right) .
\end{aligned}
$$

On the other hand, the orthogonality of $\hat{\Phi}_{a b, I}$ is assured in the above result. Therefore, the Yukawa couplings (5.16) is obtained by

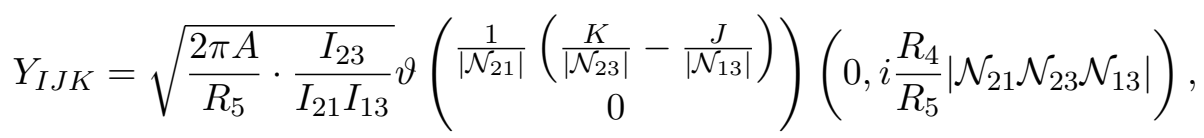

where we assume ${ }^{\exists} m \in \mathbb{Z}_{\left|\mathcal{N}_{21}\right|+\left|\mathcal{N}_{31}\right|}$ such that $K=I+J+\left|\mathcal{N}_{21}\right| m$.

The Yukawa couplings (5.17) differ from the commutative case (cf., [7]) by the overall factor only if we fix the generation numbers $\mathcal{N}_{23}, \mathcal{N}_{21}$ and $\mathcal{N}_{13}$. On the other hand, we can confirm that the Yukawa couplings (5.17) go back to the commutative case in the limit $\theta^{45} \rightarrow 0$ since the normalization constant goes back to the commutative case. ${ }^{7}$ However, the limit $\theta^{45} \rightarrow 0$ is non-trivial since the NC parameter $\theta^{45}$ remains in the Yukawa couplings, cf., $\tilde{\Gamma}^{i}:=\theta^{i j} \Gamma_{j}$ in eq. (A.9).

\footnotetext{
${ }^{7}$ The normalization constants are slightly different between the commutative case and our case. This is because the integral of 1 is normalized to obtain the area of the torus in the commutative case. In our case, the integral of $\hat{1}$ is normalized by considering the analogy of quantum mechanics (2.15). This normalization is natural from the viewpoint of the NC torus without magnetic fluxes. Therefore, if we consider the integral of 1 is normalized to obtain 1 in the commutative case, the normalization constants are the same between the commutative case and our case.
} 


\section{Conclusions and discussions}

In this paper, we performed the analysis of the chirality and the generation structures on the magnetized NC torus based on the IKKT model. In subsection 4.2, we proposed the suitable Dirac operator on the NC torus by considering noncommutative geometry. In section 5, we analyzed the zero-mode solutions of the Dirac operator we proposed. We showed that zero-mode solutions have the chirality and the generations structures. In addition, we computed the Yukawa couplings of chiral matter fields. Compared with the commutative case, the difference of the Yukawa couplings is the overall factor only. Advantages of our method are (i) we can consider geometric conditions such as periodic boundary conditions (ii) we can write down the analytic form of zero-mode solutions which can easily be compared with the commutative case. This is important to observe NC effects from the IKKT model.

When we consider the microscopic world, we compute the physical quantities through functions on spacetime like wavefunctions. On the other hand, in noncommutative geometry, we consider a function algebra on a certain space which has a NC product. For example, the star-product is a NC product in the context of deformation quantization. In the relationship between analytical mechanics and quantum mechanics, this corresponds to the replacement of the coordinate of the phase space by operators. Therefore, in the sense of noncommutative geometry, we can admit that the chirality and the generation structures of our zero-mode solutions have the physical meanings even though zero-mode solutions are written by the operators.

From a Phenomenological point of view, our results may not be new because the difference of the Yukawa couplings is the overall factor only. However, the important point of this paper is that the IKKT model can describe the string-motivated model including the NC effect. The IKKT model is considered as a non-perturbative formulation of superstring theory. Therefore, we expect that the IKKT model should describe the results of stringmotivated models known so far. From this point of view, our results are important.

We are interested in generalizations: orbifolds, complex structure + Wilson line and the origin of the magnetic flux.

The toroidal orbifolds are typical models in string phenomenology. In magnetized toroidal orbifolds, the generation structure differs from the toroidal compactifications without orbifold projections. In refs. [19, 20], the NC toroidal orbifolds are considered.

On the other hand, in general, the Yukawa couplings in the toroidal compactifications are functions of the complex structure moduli and the Wilson lines. Therefore, the values of the complex structure moduli and the Wilson lines are important to compare with the observed values (cf., [21]). Our results correspond to the case whose complex structure $\tau$ is $\tau=i R_{4} / R_{5}$. We can consider the complexification when we introduce the basic derivations. We expect that the Yukawa couplings on the magnetized NC torus are obtained by the general complex structure $\tau$ instead of $\tau=i R_{4} / R_{5}$ and the overall factor including $\tau$.

In our results, the magnetic flux played an important role. However, we introduced the magnetic flux by hand. We expect that the magnetic flux is also generated from the dynamics of the IKKT model. Recently, in refs. [22, 23], the authors showed that the 
magnetic flux may come from the tachyon condensations induced from the dynamics of D-branes and non-BPS D-branes. We expect that our results can be described by the full dynamics of the IKKT model.

Gauge selection. In this paper, we selected the axial gauge (5.1). Our method, especially the ansatz of the zero-mode solutions which are similar to the Fourier transformation, depends on the gauge selection. Therefore, we should confirm the gauge invariance of our results.

In the commutative case, the background gauge field with the fixed magnetic flux $F$ is obtained by

$$
A_{4}=-t F x^{5}, \quad A_{5}=(1-t) F x^{4},
$$

where $t \in[0,1]$. We can realize the gauge transformation from ${ }^{\forall} t_{1}$ to ${ }^{\forall} t_{2}$ by $U=$ $\exp \left[i F\left(t_{2}-t_{1}\right) x^{4} x^{5}\right]$.

On the other hand, the background gauge field on the NC torus with the fixed magnetic flux $\mathcal{F}$ is obtained by

$$
\hat{A}_{4}=-t \mathcal{F} \hat{X}^{5}, \quad \hat{A}_{5}=(1-t) \mathcal{F} \hat{X}^{4},
$$

where $t \in[0,1]$. We expect that the gauge transformation from ${ }^{\forall} t_{1}$ to ${ }^{\forall} t_{2}$ by the unitary operator, at least, $\hat{U}=\exp \left[i \alpha\left(\hat{X}^{4} \hat{X}^{5}+\hat{X}^{5} \hat{X}^{4}\right)\right]$, where $\alpha \in \mathbb{R}$. However, we can find a condition such that

$$
\left(1-t_{1} \theta^{45} \mathcal{F}\right)\left(1+\left(t_{1}-1\right) \theta^{45} \mathcal{F}\right)=\left(1-t_{2} \theta^{45} \mathcal{F}\right)\left(1+\left(t_{2}-1\right) \theta^{45} \mathcal{F}\right) \quad \text { for all } \alpha \in \mathbb{R} .
$$

If we fix the starting point $t=t_{1}$, then $t_{2}$ must be $t_{1}$ or $1-t_{1}$ since eq. (6.1) is a quadratic equation with respect to the $t_{2}$. We need to confirm whether the gauge transformations from ${ }^{\forall} t_{1}$ to ${ }^{\forall} t_{2}$ exist. This situation is the same with a gauge theory on a NC space with the star-product formulation. This is an open question. If we cannot find, different gauge backgrounds may correspond to physically different theories.

The limit: $\boldsymbol{\theta}^{\mathbf{4 5}} \rightarrow \mathbf{0}$. The zero-mode solutions (5.10) and (5.12) become those of the commutative case if we consider the limit $\theta^{45} \rightarrow 0$ and assume the operators $\left(\hat{X}^{4}, \hat{X}^{5}\right)$ correspond to the coordinate on the torus $\left(x^{4}, x^{5}\right)$. In addition, the Yukawa couplings (5.17) are the same with the commutative case in this limit. However, from the discussion in ref. [15] (or appendix A), the NC parameter remains in the effective action, e.g., the effective metric. Therefore, in terms of the effective action, the limit $\theta^{45} \rightarrow 0$ is non-trivial. This is also an open question for us.

\section{Acknowledgments}

The author would like to thank Hiroyuki Abe for helpful comments. 


\section{A Effective action of the IKKT model}

We derive the effective action of the IKKT model by considering eq. (4.2). We refer to [7] for the basic techniques. In this appendix, we consider the whole spacetime again.

The action of the IKKT model is

$$
S=-\frac{1}{g^{2}} \operatorname{Tr}\left(\frac{1}{4}\left[\hat{X}_{M}, \hat{X}_{N}\right]\left[\hat{X}^{M}, \hat{X}^{N}\right]+\frac{1}{2} \overline{\hat{\psi}} \Gamma^{M}\left[\hat{X}_{M}, \hat{\psi}\right]\right),
$$

where $M, N=0 \sim 9$. First, we consider the bosonic part of the action (A.1), i.e.,

$$
S_{b}=-\frac{1}{4 g^{2}} \operatorname{Tr}\left(\left[\hat{X}_{M}, \hat{X}_{N}\right]\left[\hat{X}^{M}, \hat{X}^{N}\right]\right)
$$

We introduced the gauge field as a fluctuation (4.2). By substituting,

$$
S_{b}=-\frac{1}{4 g^{2}} \operatorname{Tr}\left(\eta_{I K} \eta_{J L}\left[\hat{X}_{\mathrm{bg}}^{I}+\theta^{I M} \hat{A}_{M}, \hat{X}_{\mathrm{bg}}^{J}+\theta^{J M} \hat{A}_{M}\right]\left[\hat{X}_{\mathrm{bg}}^{K}+\theta^{K N} \hat{A}_{N}, \hat{X}_{\mathrm{bg}}^{L}+\theta^{L N} \hat{A}_{N}\right]\right) .
$$

For concreteness, we consider the $\mathrm{U}(N)$ gauge group. $\left(U_{a}\right)_{j}^{i}=\delta_{a i} \delta_{a j}$ and $\left(e_{a b}\right)_{i j}=\delta_{a i} \delta_{b j}$ can be selected as the basis of the Lie algebra. Accordingly, the trace of the action (A.3) is defined on the operator space and the gauge group. Then, we can expand the gauge field and the fermions in the adjoint representation as

$$
\begin{aligned}
\hat{A}_{M} & =\hat{B}_{M}+\hat{W}_{M}=\hat{B}_{M}^{a} U_{a}+\hat{W}_{M}^{a b} e_{a b}, \\
\hat{\psi} & =\hat{\chi}+\hat{\Psi}=\hat{\chi}^{a} U_{a}+\hat{\Psi}^{a b} e_{a b} .
\end{aligned}
$$

Let us define

$$
\begin{array}{rlrl}
\partial_{M} & =-i\left(\theta^{-1}\right)_{M N}\left[\hat{X}_{\mathrm{bg}}^{N}, \cdot\right] & & (\text { Partial derivatives }), \\
\hat{F}_{M N} & =\partial_{M} \hat{B}_{N}-\partial_{N} \hat{B}_{M}-i\left[\hat{B}_{M}, \hat{B}_{N}\right] & & \text { (Field strength of the U(1) gauge group), } \\
D_{M} \hat{W}_{N} & =\partial_{M} \hat{W}_{N}-i\left[\hat{B}_{M}, \hat{W}_{N}\right] & & \text { (Covariant derivatives } \\
& & \text { with respect to the U(1) gauge group), } \\
G^{M N} & =\theta^{M I} \theta^{N J} \eta_{I J} & & \text { (Effective metric) }
\end{array}
$$

then eq. (A.3) is rewritten as

$$
\begin{aligned}
S_{b}= & \frac{1}{4 g^{2}} \operatorname{Tr}\left(\left(\hat{F}_{M N}-\left(\theta^{-1}\right)_{M N}\right)\left(\hat{F}^{M N}-\left(\theta^{-1}\right)^{M N}\right)-\left[\hat{W}_{M}, \hat{W}_{N}\right]\left[\hat{W}^{M}, \hat{W}^{N}\right]\right) \\
& \left.+\frac{1}{2 g^{2}} \operatorname{Tr}\left(D_{M} \hat{W}_{N} D^{M} \hat{W}^{N}-D_{M} \hat{W}_{N} D^{N} \hat{W}^{M}-i\left(\hat{F}_{M N}-\left(\theta^{-1}\right)_{M N}\right)\left[\hat{W}^{M}, \hat{W}^{N}\right]\right)\right),
\end{aligned}
$$

where the indices are contracted by the effective metric, and we used the cyclic property of the trace. 
Next, we introduce the Abelian magnetic flux on the extra-dimensional space $(i=4,5)$, i.e.,

$$
\hat{B}_{i}^{a}=<\hat{B}_{i}^{a}>+\hat{C}_{i}^{a}, \quad \hat{W}_{i}^{a b}=\hat{\Phi}_{i}^{a b},
$$

where $\hat{C}_{i}^{a}$ and $\hat{\Phi}_{i}^{a b}$ are fluctuations around the Abelian magnetic flux background (we set $<\hat{W}_{i}^{a b}>=0$ ). By substituting the background (A.6), eq. (A.3) is rewritten as

$$
S_{b}=-\frac{1}{2 g^{2}} \operatorname{Tr}\left(\hat{\Phi}_{i} D_{\mu} D^{\mu} \hat{\Phi}^{i}+\hat{\Phi}_{j} \tilde{D}_{i} \tilde{D}^{i} \hat{\Phi}^{j}\right)-\frac{i}{2 g^{2}}\left(<\hat{F}_{i j}^{a}>-<\hat{F}_{i j}^{b}>\right) \hat{\Phi}^{i, a b} \hat{\Phi}^{j, b a}+S_{b, \text { other }}
$$

where

$$
\tilde{D}_{i} \hat{\Phi}_{j}^{a b}:=\partial_{i} \hat{\Phi}_{j}^{a b}-i<\hat{B}_{i}^{a}>\hat{\Phi}_{j}^{a b}+i \hat{\Phi}_{j}^{a b}<\hat{B}_{i}^{b}>,
$$

and we used the cyclic property of the trace. $S_{b, \text { other }}$ contains irrelevant terms for our main discussions.

In eq. (A.7), we can see the Laplacian on the extra-dimensional space, i.e.,

$$
\Delta_{6 d}:=-G^{i j} \tilde{D}_{i} \tilde{D}_{j}=\sum_{i}\left[\hat{X}^{i},\left[\hat{X}^{i}, \cdot\right]\right] .
$$

Similarly, we can obtain the fermionic part of the effective action

$$
S_{f}=-\frac{1}{2 g^{2}} \operatorname{Tr}\left(i \overline{\hat{\Psi}} \tilde{\Gamma}^{\mu} D_{\mu} \hat{\Psi}+i \overline{\hat{\Psi}} \tilde{\Gamma}^{i} \tilde{D}_{i} \hat{\Psi}+\overline{\hat{\Psi}} \tilde{\Gamma}^{i}\left[\hat{\Phi}_{i}, \hat{\Psi}\right]\right)+S_{f, \text { other }}
$$

where the indices are contracted by the Minkowski metric and $\tilde{\Gamma}^{\mu}:=\theta^{\mu \nu} \Gamma_{\nu}$, and $S_{f \text {,other }}$ contains irrelevant terms for our main discussions.

Open Access. This article is distributed under the terms of the Creative Commons Attribution License (CC-BY 4.0), which permits any use, distribution and reproduction in any medium, provided the original author(s) and source are credited.

\section{References}

[1] N. Ishibashi, H. Kawai, Y. Kitazawa and A. Tsuchiya, A large $N$ reduced model as superstring, Nucl. Phys. B 498 (1997) 467 [hep-th/9612115] [INSPIRE].

[2] A. Chatzistavrakidis, H. Steinacker and G. Zoupanos, Intersecting branes and a standard model realization in matrix models, JHEP 09 (2011) 115 [arXiv:1107.0265] [INSPIRE].

[3] H.C. Steinacker and J. Zahn, An extended standard model and its Higgs geometry from the matrix model, PTEP 2014 (2014) 083B03 [arXiv: 1401.2020] [INSPIRE].

[4] H. Aoki, J. Nishimura and A. Tsuchiya, Realizing three generations of the Standard Model fermions in the type IIB matrix model, JHEP 05 (2014) 131 [arXiv: 1401.7848] [INSPIRE].

[5] H. Aoki, J. Nishimura and Y. Susaki, Finite-matrix formulation of gauge theories on a non-commutative torus with twisted boundary conditions, JHEP 04 (2009) 055 [arXiv: 0810.5234] [INSPIRE]. 
[6] A. Connes, M.R. Douglas and A.S. Schwarz, Noncommutative geometry and matrix theory: Compactification on tori, JHEP 02 (1998) 003 [hep-th/9711162] [INSPIRE].

[7] D. Cremades, L.E. Ibáñez and F. Marchesano, Computing Yukawa couplings from magnetized extra dimensions, JHEP 05 (2004) 079 [hep-th/0404229] [INSPIRE].

[8] P. Austing and J.F. Wheater, The Convergence of Yang-Mills integrals, JHEP 02 (2001) 028 [hep-th/0101071] [INSPIRE].

[9] P. Austing and J.F. Wheater, Convergent Yang-Mills matrix theories, JHEP 04 (2001) 019 [hep-th/0103159] [INSPIRE].

[10] A. Konechny and A.S. Schwarz, Introduction to M(atrix) theory and noncommutative geometry, Phys. Rept. 360 (2002) 353 [hep-th/0012145] [INSPIRE].

[11] J. Dixmier, Représentations irrèducibles des algèbres de Lie nilpotentes, An. Acad. Brasil Ci. 35 (1963) 491.

[12] D. Quillen, On the endomorphism ring of a simple module over an enveloping algebra, Proc. Am. Math. Soc. 21 (1969) 171.

[13] M.A. Rieffel, Projective modules over higher-dimensional non-commutative tori, Can. J. Math. XL (1988) 257.

[14] Y. Tenjinbayashi, H. Igarashi and T. Fujiwara, Dirac operator zero-modes on a torus, Annals Phys. 322 (2007) 460 [hep-th/0506259] [INSPIRE].

[15] H. Steinacker, Emergent Gravity from Noncommutative Gauge Theory, JHEP 12 (2007) 049 [arXiv: 0708.2426] [INSPIRE].

[16] N. Seiberg and E. Witten, String theory and noncommutative geometry, JHEP 09 (1999) 032 [hep-th/9908142] [INSPIRE].

[17] J. Nishimura and A. Tsuchiya, Realizing chiral fermions in the type IIB matrix model at finite N, JHEP 12 (2013) 002 [arXiv:1305.5547] [INSPIRE].

[18] D. Mumford, Tata lectures on theta I, Birkhäuser, Boston U.S.A. (1983).

[19] A. Konechny and A.S. Schwarz, Compactification of M(atrix) theory on noncommutative toroidal orbifolds, Nucl. Phys. B 591 (2000) 667 [hep-th/9912185] [INSPIRE].

[20] S. Walters, Toroidal orbifolds of $\mathbb{Z}_{3}$ and $\mathbb{Z}_{6}$ symmetries of noncommutative tori, Nucl. Phys. B 894 (2015) 496 [INSPIRE].

[21] H. Abe, T. Kobayashi, H. Ohki, A. Oikawa and K. Sumita, Phenomenological aspects of $10 D$ SYM theory with magnetized extra dimensions, Nucl. Phys. B 870 (2013) 30 [arXiv: 1211.4317] [INSPIRE].

[22] T. Asakawa and S. Matsuura, Spherical D-brane by Tachyon Condensation, PTEP 2018 (2018) 033B01 [arXiv:1703.10352] [INSPIRE].

[23] T. Asakawa, G. Ishiki, T. Matsumoto, S. Matsuura and H. Muraki, Commutative Geometry for Non-commutative D-branes by Tachyon Condensation, PTEP 2018 (2018) 063B04 [arXiv: 1804.00161] [INSPIRE]. 